\title{
Evidence for VHE $\gamma$-ray emission from the distant BL Lac PG 1553+113
}

F. Aharonian ${ }^{1}$, A. G. Akhperjanian ${ }^{2}$, A. R. Bazer-Bachi ${ }^{3}$, M. Beilicke ${ }^{4}$, W. Benbow ${ }^{1}$, D. Berge ${ }^{1}$, K. Bernlöhr $^{1,5}$, C. Boisson ${ }^{6}$, O. Bolz ${ }^{1}$, V. Borrel ${ }^{3}$, I. Braun ${ }^{1}$, F. Breitling ${ }^{5}$, A. M. Brown ${ }^{7}$, R. Bühler ${ }^{1}$, S. Carrigan ${ }^{1}$, P. M. Chadwick ${ }^{7}$, L.-M. Chounet ${ }^{8}$, R. Cornils ${ }^{4}$, L. Costamante ${ }^{1,20}$, B. Degrange ${ }^{8}$, H. J. Dickinson ${ }^{7}$, A. Djannati-Atai ${ }^{9}$, L. O' $^{\prime}$ C. Drury ${ }^{10}$, G. Dubus ${ }^{8}$, K. Egberts ${ }^{1}$, D. Emmanoulopoulos ${ }^{11}$, P. Espigat ${ }^{9}$, F. Feinstein ${ }^{12}$, G. Fontaine ${ }^{8}$, S. Funk ${ }^{1}$, Y. A. Gallant ${ }^{12}$, B. Giebels ${ }^{8}$, J. F. Glicenstein ${ }^{14}$, P. Goret ${ }^{14}$, C. Hadjichristidis ${ }^{7}$, D. Hauser ${ }^{1}$, M. Hauser ${ }^{11}$, G. Heinzelmann ${ }^{4}$, G. Henri ${ }^{13}$, G. Hermann ${ }^{1}$, J. A. Hinton ${ }^{1,11}$, W. Hofmann ${ }^{1}$, M. Holleran ${ }^{15}$, D. Horns ${ }^{1}$, A. Jacholkowska ${ }^{12}$, O. C. de Jager ${ }^{15}$, B. Khélifi ${ }^{8,1}$, Nu. Komin ${ }^{5}$, A. Konopelko ${ }^{5}$, I. J. Latham ${ }^{7}$, R. Le Gallou ${ }^{7}$, A. Lemière ${ }^{9}$,

M. Lemoine-Goumard ${ }^{8}$, T. Lohse ${ }^{5}$, J. M. Martin ${ }^{6}$, O. Martineau-Huynh ${ }^{16}$, A. Marcowith ${ }^{3}$, C. Masterson ${ }^{1,20}$, T. J. L. McComb ${ }^{7}$, M. de Naurois ${ }^{16}$, D. Nedbal ${ }^{17}$, S. J. Nolan ${ }^{7}$, A. Noutsos ${ }^{7}$, K. J. Orford ${ }^{7}$, J. L. Osborne ${ }^{7}$, M. Ouchrif ${ }^{16,20}$, M. Panter ${ }^{1}$, G. Pelletier ${ }^{13}$, S. Pita ${ }^{9}$, G. Pühlhofer ${ }^{11}$, M. Punch ${ }^{9}$, B. C. Raubenheimer ${ }^{15}$, M. Raue ${ }^{4}$, S. M. Rayner ${ }^{7}$, A. Reimer ${ }^{18}$, O. Reimer ${ }^{18}$, J. Ripken ${ }^{4}$, L. Rob ${ }^{17}$, L. Rolland ${ }^{16}$, G. Rowell ${ }^{1}$, V. Sahakian ${ }^{2}$, L. Saugé $^{13}$, S. Schlenker ${ }^{5}$, R. Schlickeiser ${ }^{18}$, C. Schuster ${ }^{18}$, U. Schwanke ${ }^{5}$, M. Siewert ${ }^{18}$, H. Sol ${ }^{6}$, D. Spangler ${ }^{7}$, R. Steenkamp ${ }^{19}$, C. Stegmann ${ }^{5}$, G. Superina ${ }^{8}$, J.-P. Tavernet ${ }^{16}$, R. Terrier ${ }^{9}$, C. G. Théoret ${ }^{9}$, M. Tluczykont ${ }^{8,20}$, C. van Eldik $^{1}$, G. Vasileiadis ${ }^{12}$, C. Venter ${ }^{15}$, P. Vincent ${ }^{16}$, H. J. Völk ${ }^{1}$, S. J. Wagner ${ }^{11}$, and M. Ward ${ }^{7}$

(Affiliations can be found after the references)

Received 14 December 2005 / Accepted 19 January 2006

\section{ABSTRACT}

The high-frequency peaked BL Lac PG 1553+113 was observed in 2005 with the HESS stereoscopic array of imaging atmospheric-Cherenkov telescopes in Namibia. Using the HESS standard analysis, an excess was measured at the $4.0 \sigma$ level in these observations (7.6 hours live time). Three alternative, lower-threshold analyses yield $>5 \sigma$ excesses. The observed integral flux above $200 \mathrm{GeV}$ is $\left(4.8 \pm 1.3_{\text {stat }} \pm 1.0_{\text {syst }}\right) \times$ $10^{-12} \mathrm{~cm}^{-2} \mathrm{~s}^{-1}$, and shows no evidence for variability. The measured energy spectrum is characterized by a very soft power law (photon index of $\Gamma=4.0 \pm 0.6$ ). Although the redshift of PG $1553+113$ is unknown, there are strong indications that it is greater than $z=0.25$ and possibly larger than $z=0.78$. The observed spectrum is interpreted in the context of VHE $\gamma$-ray absorption by the Extragalactic Background Light, and is used to place an upper limit on the redshift of $z<0.74$.

Key words. galaxies: active - BL Lacertae objects: individual: PG 1553+113 - gamma rays: observations

\section{Introduction}

PG $1553+113$ was discovered in the Palomar-Green survey of UV-excess stellar objects (Green et al. 1986). It is classified as a BL Lac object based on its featureless spectrum (Miller \& Green 1983; Falomo \& Treves 1990) and its significant $\left(m_{p}=13.2-15.0\right)$ optical variability (Miller et al. 1988). PG $1553+113$ is well studied from the radio to the X-ray regime, and has been the subject of several multi-wavelength observation campaigns. In X-rays it has been detected by multiple observatories, with energy spectra measured by both BeppoSAX (Donato et al. 2005) and XMM Newton (Perlman et al. 2005). Based on its broad-band spectral energy distribution (SED), PG 1553+113 is now classified as a highfrequency peaked BL Lac (Giommi et al. 1995), similar to essentially all of the AGN detected at VHE (very high energy; $>100 \mathrm{GeV}$ ) energies.
The redshift of PG $1553+113$ was initially determined to be $z=0.36$ (Miller \& Green 1983). However, it was later claimed that this value is flawed as it was based on a spurious emission line, caused by a bright spot in the International Ultraviolet Explorer image, that was misidentified as Lyman$\alpha$ (Falomo \& Treves 1990). To date no emission or absorption lines have been measured despite more than ten observation campaigns with optical instruments, including two with the 8-meter VLT telescopes (Wagner 2005). Estimates of the redshift can also be made using photometric observations. No host galaxy was resolved in Hubble Space Telescope (HST) images of PG $1553+113$ taken during the HST survey of 110 BL Lac objects (Scarpa et al. 2000). Approximately $80 \%$ of these BL Lacs (88) have known redshifts, of which all 39 with $z<0.25$ and 21 of the 28 with $0.25<z<0.6$ have their hosts resolved (Sbarufatti et al. 2005). The HST results were recently 
used to set a lower limit of $z>0.78$ (Sbarufatti et al. 2005) for PG $1553+113$. This redshift limit is based on the assumption of the nucleus being present in a typical host galaxy, and general correlation between photometrically determined redshifts and those determined spectroscopically. However, a departure from this correlation, or the possibility of an atypical host galaxy is not unexpected. The possibility of such a large redshift is of critical importance to VHE observations due to the absorption of VHE photons (Stecker et al. 1992; Hauser \& Dwek 2001) by pair-production on the Extragalactic Background Light (EBL). This absorption, which is energy dependent and increases strongly with redshift, distorts the VHE energy spectra observed from distant objects, and may even render the VHE detection of very distant objects impossible.

Although the redshift of PG $1553+113$ is essentially unknown, it is viewed as a promising candidate for detection as a VHE emitter (Costamante \& Ghisellini 2002) based on its SED. However, it has not been previously detected in the VHE regime. Only flux level upper limits have been reported by the Whipple collaboration (de la Calle Perez et al. 2003) and the Milagro group (Williams 2004). Here, evidence for $>200 \mathrm{GeV}$ $\gamma$-ray emission from PG $1553+113$ is presented, based on observations made with the HESS (High Energy Stereoscopic System) experiment. The data are used to set an upper limit on the source redshift.

\section{HESS detector and observations}

The HESS experiment is a stereoscopic system of four imaging atmospheric-Cherenkov telescopes located in Namibia. HESS is designed to search for astrophysical $\gamma$-ray emission above $\sim 100 \mathrm{GeV}$, with a sensitivity $(5 \sigma$ in $25 \mathrm{~h}$ for a $1 \%$ Crab Nebula flux source at $20^{\circ}$ zenith angle) that allows for the detection of VHE emission from objects such as PG $1553+113$ at previously undetectable flux levels. More details on HESS can be found in Hinton (2004).

The HESS observations of PG $1553+113$ were made in May and August 2005. A total of 22 runs ( $28 \mathrm{~min}$ each) were taken, yielding a total exposure of $9.6 \mathrm{~h}$. Seventeen of these runs pass selection criteria which remove data for which the weather conditions were poor or the hardware was not functioning properly. The total exposure of the quality selected data set is $7.6 \mathrm{~h}$ live time, and the mean zenith angle of these observations is $40^{\circ}$. All these data were taken using Wobble mode, i.e. the source direction is positioned $\pm 0.5^{\circ}$ relative to the center of the field of view (f.o.v.) of the camera during observations, which allows for both on-source observations and simultaneous estimation of the background induced by charged cosmic rays.

\section{Analysis technique}

The data passing the run selection criteria are calibrated as detailed in Aharonian et al. (2004), and the event reconstruction and background rejection (i.e. event selection criteria) are performed as described in Aharonian et al. (2005a), with some minor improvements discussed in Aharonian et al. (2005b). On-source data are taken from a circular region of radius $\theta_{\text {cut }}$
Table 1. The selection cuts applied to the data for the HESS standard analysis and those applied for the spectrum determination in this article. Cuts keeping only events with MRSL and MRSW greater than $-2.0 \sigma$ are used in both cases. Only images passing the distance and size cuts are used in the analysis, and a minimum of two such images are required.

\begin{tabular}{cccccc}
\hline \hline Cut & MRSL & MRSW & $\theta_{\text {cut }}^{2}$ & Size & $\begin{array}{c}\text { Dist. } \\
\text { Type }\end{array}$ \\
$\begin{array}{c}\max \\
{[\sigma]}\end{array}$ & $\begin{array}{c}\max \\
{[\sigma]}\end{array}$ & $\begin{array}{c}\max \\
{\left[\mathrm{deg}^{2}\right]}\end{array}$ & $\begin{array}{c}\min \\
{[\mathrm{PE}]}\end{array}$ & $\begin{array}{c}\max \\
{[\mathrm{mrad}]}\end{array}$ \\
\hline Standard & 2.0 & 0.9 & 0.0125 & 80 & 35 \\
Spectrum & 1.3 & 0.9 & 0.02 & 40 & 35 \\
\hline
\end{tabular}

centered on the source. The background is estimated using events from a number of equal-size off-source regions offset by the same distance, relative to the center of the f.o.v., in the sky as the on-source region (for more details see Aharonian et al. 2005c). The significance of any excess is calculated following the method of Eq. (17) in Li \& Ma (1983).

Table 1 shows the cuts on mean reduced scaled width (MRSW) and length (MRSL) parameters (Aharonian et al. 2005a), on $\theta^{2}$ (the square of the angular difference between the reconstructed shower direction and the source position), on individual image size (in photoelectrons; $\mathrm{PE}$ ), and on the distance of the image center of gravity from the center of the f.o.v., used in this analysis. The standard HESS analysis cuts shown in Table 1 are optimized a priori using Monte Carlo $\gamma$-ray simulations and unrelated off-source data to yield the maximum expected significance per hour of observation for a weak (10\% Crab flux source with photon index $\Gamma=2.6$ ) source. Generally, the significance expected (and observed) is not strongly dependent on the exact values of the cuts. However, the size cut increases the energy threshold of the analysis, therefore lower values are more appropriate for a steep spectrum source.

\section{Results}

Table 2 shows the results from the analysis of the HESS observations of PG $1553+113$. Using the standard cuts, an excess of 83 events $(4.0 \sigma)$ is observed from the direction of PG 1553+113. Figure 1 shows the on-source and normalized off-source distributions of $\theta^{2}$ for the HESS observations. The background is approximately flat in $\theta^{2}$ as expected, and there is an excess at small values of $\theta^{2}$ corresponding to the observed signal. The shape of this excess is consistent with that expected from a point source given the HESS point-spread function.

Assuming a power-law spectrum $\left(\mathrm{d} N / \mathrm{d} E \sim E^{-\Gamma}\right)$ with a photon index of $\Gamma=4.0$ (justified later), the observed integral flux above $200 \mathrm{GeV}$ is $I(>200 \mathrm{GeV})=\left(4.8 \pm 1.3_{\text {stat }} \pm 1.0_{\text {syst }}\right) \times$ $10^{-12} \mathrm{~cm}^{-2} \mathrm{~s}^{-1}$. This corresponds to $\sim 2 \%$ of $I(>200 \mathrm{GeV})$ determined by HESS for the Crab Nebula. The flux is well below all previously published upper limits for PG $1553+113$. No evidence for variability is found as a fit to the nightly integral flux versus time is consistent with being constant $\left(\chi^{2}=3.7,7\right.$ degrees of freedom). Additionally, quick-look results provided by 
Table 2. Results of the HESS observations of PG $1553+113$ using both the standard analysis cuts and the lower-threshold spectral analysis cuts. Shown are the post-cuts energy threshold at the mean zenith angle of observations $\left(40^{\circ}\right)$, the number of on-source and off-source events passing the cuts, the normalization for the off-source events, the observed excess $(\Delta)$ from PG $1553+113$ and the significance (S) of the excess. The significance resulting from the spectral analysis cuts is subject to a small trials factor. Therefore only the significance resulting from the HESS standard analysis should be considered.

\begin{tabular}{ccccccc}
\hline \hline $\begin{array}{c}\text { Cut } \\
\text { Type }\end{array}$ & $\begin{array}{c}E_{\mathrm{th}} \\
{[\mathrm{GeV}]}\end{array}$ & On & Off & Norm & $\Delta$ & $\begin{array}{c}\mathrm{S} \\
{[\sigma]}\end{array}$ \\
\hline Standard & 310 & 457 & 4126 & 0.0907 & 83 & 4.0 \\
Spectrum & 230 & 1807 & 12753 & 0.1236 & 230 & 5.3 \\
\hline
\end{tabular}

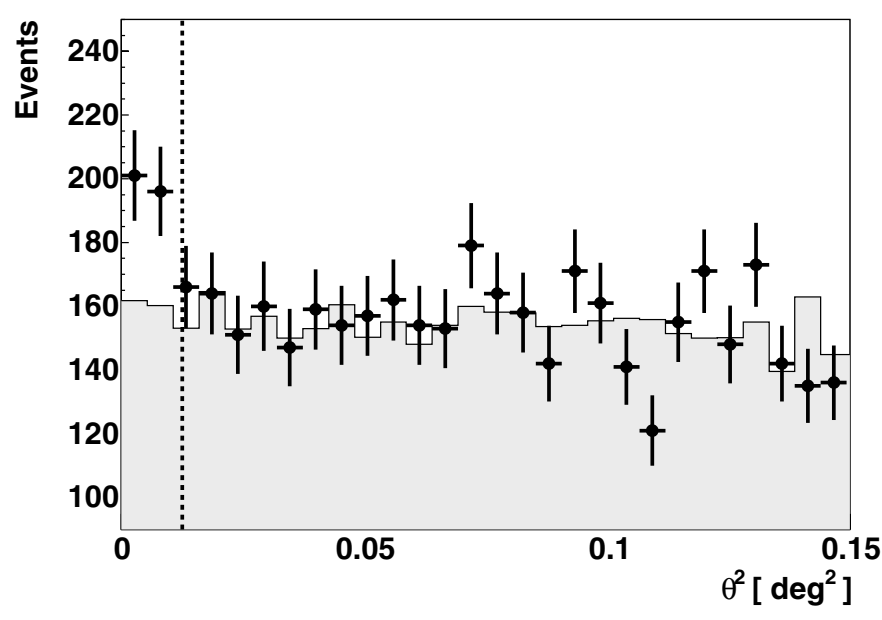

Fig. 1. The distribution of $\theta^{2}$ for on-source events (points) and normalized off-source events (shaded) from observations of PG 1553+113. The dashed line represents the cut on $\theta^{2}$ applied to the data.

the RXTE/ASM team show no indication of flaring behavior at $\mathrm{X}$-ray energies (2 to $10 \mathrm{keV}$ ), suggesting that PG $1553+113$ was not in a high-emission state.

Given the low number of excess events found using the HESS standard analysis, the determination of an energy spectrum is not possible. Therefore, a set of lower-threshold cuts were generated to increase the selection efficiency for lowenergy $\gamma$-rays, allowing for a spectral determination. The cuts were generated using the exact same procedure as for the standard analysis cuts, differing only in that they were optimized for a $1 \%$ Crab flux $(>100 \mathrm{GeV})$ source with photon index $\Gamma=5.0$. Using these cuts (also shown in Table 1) an excess of 230 events $(5.3 \sigma)$ from the direction of PG $1553+113$ is found. The significance of this excess is clearly subject to a small trials factor and is therefore reduced. To be conservative, only the significance of the standard analysis should be considered. The energy spectrum determined from the data set, using the lowthreshold analysis, is shown in Fig. 2. The best $\chi^{2}$ fit of a power law to these data yields a photon index $\Gamma=4.0 \pm 0.6_{\text {stat }}$, and a $\chi^{2}$ of 1.8 for 2 degrees of freedom. The systematic error on the photon index is small compared to the statistical error.

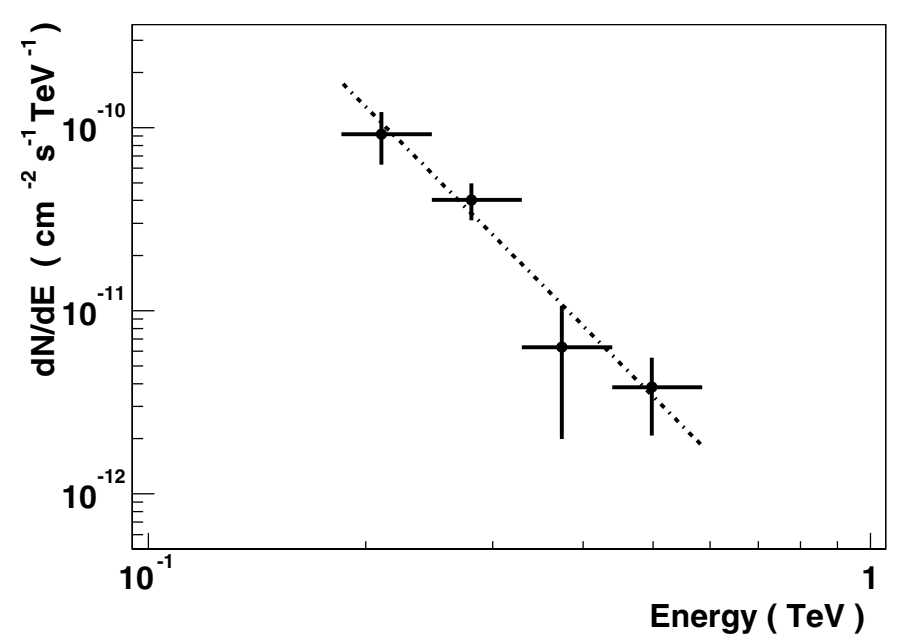

Fig. 2. The energy spectrum of PG $1553+113$. The horizontal error bars indicate the energy bin size. The dashed line represents the best $\chi^{2}$ fit of a power law.

It should be noted that the integral flux (again assuming $\Gamma=4.0)$ determined with the "spectrum" cuts, $I(>200 \mathrm{GeV})=$ $\left(5.8 \pm 1.1_{\text {stat }} \pm 1.2_{\text {syst }}\right) \times 10^{-12} \mathrm{~cm}^{-2} \mathrm{~s}^{-1}$, is consistent with the flux obtained using the standard cuts. This indicates that the increased excess found with these cuts is indeed expected and not the result of over-optimization or statistical fluctuations. In addition, a cross check was performed on the same data as published by HESS on PKS 2005-489 (Aharonian et al. 2005b). Here an analysis using the spectraldetermination cuts results in the same spectrum $(\Gamma=4.0)$ as previously published, as well as the increase of the detected excess by similar factor as for PG $1553+113$. Finally, an alternative analysis using an independent calibration and two different event reconstruction methods (de Naurois et al. 2003; Lemoine-Goumard \& Degrange 2004) verifies the observed signal ( $>5 \sigma$ excess) and yields compatible spectra.

\section{Discussion}

PG $1553+113$ possibly represents the most distant BL Lac object detected at VHE energies. Therefore, the observed VHE spectrum is expected to be affected by EBL absorption. Unfortunately the unknown redshift of this object does not allow its VHE spectrum to be used in constraining the poorly-measured EBL flux density (see e.g. Aharonian et al. 2005d). However, assuming a redshift allows the determination of the spectrum intrinsic to the BL Lac (i.e. with the effect of EBL absorption removed). The intrinsic flux is related to the observed flux by $F_{\text {int }}=\mathrm{e}^{\tau(z, E)} F_{\text {obs }}$, where $\tau(z, E)$ is the optical depth due to pair production $\left(\gamma_{\mathrm{VHE}} \gamma_{\mathrm{EBL}} \rightarrow \mathrm{e}^{+} \mathrm{e}^{-}\right)$. Assuming that the main uncertainty arises from the overall normalization of the EBL density, this optical depth can be expressed as $\tau(z, E)=f \tau_{0}(z, E)$, where $\tau_{0}(z, E)$ is calculated using a reference EBL-density model, and $f$ is a scaling factor for this density. At energies relevant to the HESS observations, $\tau(z, E)$ increases with both redshift and energy. Therefore, a larger redshift, or higher EBL flux density, causes a stronger absorption 


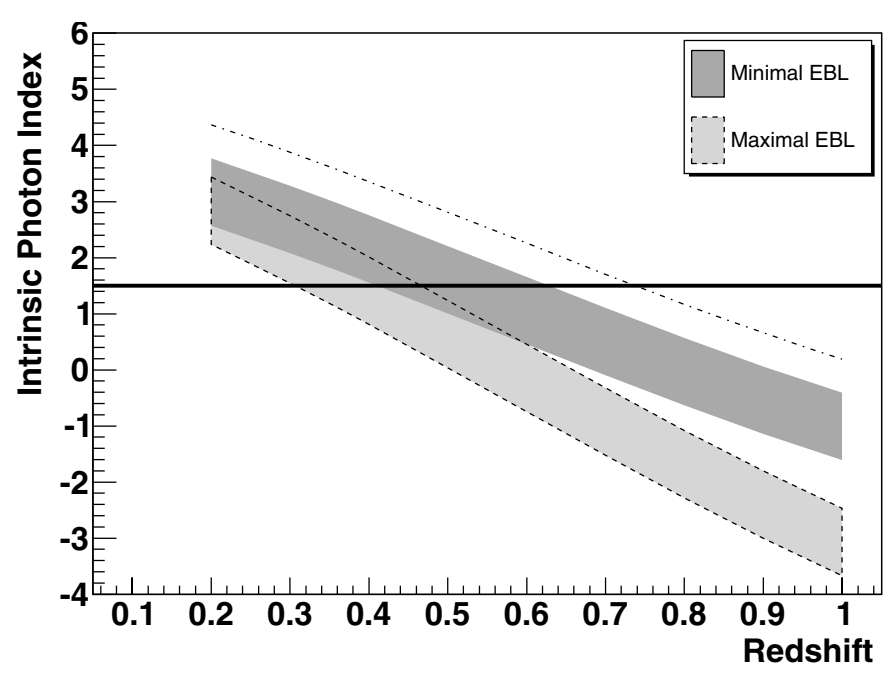

Fig. 3. The intrinsic photon index of PG $1553+113$, with $1 \sigma$ statistical error contours, versus redshift for the cases of minimal and maximal EBL absorption. An intrinsic photon index below the horizontal line $\left(\Gamma_{\text {int }}=1.5\right)$ is not considered realistic. The uppermost (dashed-dotted) curve is the sum of the intrinsic photon index and $2 \sigma$ statistical error for the case of minimal EBL absorption.

of higher-energy $\gamma$-rays, resulting in a harder intrinsic spectrum $\left(F_{\text {int }} \propto E^{-\Gamma_{\text {int }}}\right)$. The EBL density model of Primack et al. (2001) is chosen here since it includes the effects of galaxy evolution which become important at redshifts greater than $\sim 0.2$. This model's fluxes are above recently published upper limits (Aharonian et al. 2005d). However, scaling its values by $f=$ 0.85 approximately traces these limits ${ }^{1}$ and is therefore considered a maximal EBL flux density. Given that the EBL flux density is poorly-known, a minimal EBL scenario is also considered. Here, the original model is scaled by $f=0.6$, approximately tracing the lower limits on the EBL flux density provided by galaxy counts (Madau \& Pozzetti 2000). Using these parameterizations, the intrinsic spectrum of PG $1553+113$ is calculated for different redshift values. Figure 3 shows the intrinsic photon index $\left(\Gamma_{\text {int }}\right)$ versus redshift. Assuming the intrinsic photon index of the BL Lac is not harder than $\Gamma_{\text {int }}=1.5$ (see Aharonian et al. 2005d for arguments supporting this assumption), an upper limit on the redshift of PG $1553+113$ of $z<0.74$ is determined using the minimal EBL. Above this redshift, where $\Gamma_{\text {int }}=0.3 \pm 0.6$, the sum of $\Gamma_{\text {int }}$ and twice its statistical error is harder than 1.5. Clearly, the redshift upper limit is smaller if a higher EBL flux density is assumed. This is the first time that a VHE observation is used to usefully constrain the redshift of an astrophysical object. Future observations of PG 1553+113 should allow for a more precise determination of the photon index, possibly resulting in a stronger redshift constraint.

Acknowledgements. The support of the Namibian authorities and of the University of Namibia in facilitating the construction and operation of HESS is gratefully acknowledged, as is the support by the German Ministry for Education and Research (BMBF), the

${ }^{1}$ A different model and hence scaling factor is used in Aharonian et al. (2005d).
Max-Planck-Society, the French Ministry for Research, the CNRSIN2P3 and the Astroparticle Interdisciplinary Programme of the CNRS, the UK Particle Physics and Astronomy Research Council (PPARC), the IPNP of the Charles University, the South African Department of Science and Technology and National Research Foundation, and by the University of Namibia. We appreciate the excellent work of the technical support staff in Berlin, Durham, Hamburg, Heidelberg, Palaiseau, Paris, Saclay, and in Namibia in the construction and operation of the equipment.

\section{References}

Aharonian, F., Akhperjanian, A. G., Aye, K.-M., et al. (HESS Collaboration) 2004, Astropart. Phys., 22, 109

Aharonian, F., Akhperjanian, A. G., Aye, K.-M., et al. (HESS Collaboration) 2005a, A\&A, 430, 865

Aharonian, F., Akhperjanian, A. G., Aye, K.-M., et al. (HESS Collaboration) 2005b, A\&A, 436, L17

Aharonian, F., Akhperjanian, A. G., Bazer-Bachi, A. R., et al. (HESS Collaboration) 2005c, A\&A, 441, 465

Aharonian, F., et al. (HESS Collaboration) 2005d, submitted for publication [arXiv: astro-ph/0508073]

Costamante, L., \& Ghisellini, G. 2002, A\&A, 384, 56

de la Calle Perez, I., Bond, I. H., Boyle, P. J., et al. 2003, ApJ, 599 , 909

de Naurois, M., Guy, J., Djannati-Ataï, A., et al. 2003, Proc. of the 28th ICRC (Tsukuba), 2907

Donato, D., Sambruna, R. M., \& Gliozzi, M. 2005, A\&A, 433, 1163

Falomo, R., \& Treves, A. 1990, PASP, 102, 1120

Giommi, P., Ansari, S. G., \& Micol, A. 1995, A\&AS, 109, 267

Green, R. F., Schmidt, M., \& Liebert, J. 1986, ApJS, 61, 305

Hauser, M. G., \& Dwek, E. 2001, ARA\&A, 39, 249

Hinton, J. 2004, New Astron. Rev., 48, 331

Lemoine-Goumard, M., \& Degrange, B. 2004, AIP Conf. Proc., 745, 697

Li, T., \& Ma, Y. 1983, ApJ, 272, 317

Miller, H. R., Carini, M. T., Gaston, B. J., et al. 1988, Proc. of the IUE Symp. (Greenbelt), 303

Miller, H. R., \& Green, R. F. 1983, BAAS, 15, 957

Perlman, E. S., Madejski, G., Georganopoulos, M., et al. 2005, ApJ, 625,727

Madau, P., \& Pozzetti, L. 2000, MNRAS, 312, L9

Primack, J. R., Somerville, R. S., Bullock, J. S., et al. 2001, AIP Conf. Proc., 558, 463

Sbarufatti, B., Treves, A., \& Falomo, R. 2005, ApJ, 635, 173

Scarpa, R., Urry, C. M., Falomo, R., et al. 2000, ApJ, 532, 740

Stecker, F. W., de Jager, O. C., \& Salamon, M. H. 1992, ApJ, 390, 49

Wagner, S. 2005, Private Communication

Williams, D. 2004, AIP Conf. Proc., 745, 499

1 Max-Planck-Institut für Kernphysik, Heidelberg, Germany e-mail: Wystan. Benbow@mpi-hd.mpg.de

2 Yerevan Physics Institute, Armenia

3 Centre d'Étude Spatiale des Rayonnements, CNRS/UPS, Toulouse, France

${ }^{4}$ Universität Hamburg, Institut für Experimentalphysik, Germany

5 Institut für Physik, Humboldt-Universität zu Berlin, Germany

${ }^{6}$ LUTH, UMR 8102 du CNRS, Observatoire de Paris, Section de Meudon, France

7 University of Durham, Department of Physics, UK 
${ }^{8}$ Laboratoire Leprince-Ringuet, IN2P3/CNRS, École Polytechnique, Palaiseau, France

9 APC, UMR 7164 (CNRS, Université Paris VII, CEA, Observatoire de Paris), Paris, France

e-mail: santiago.pita@cdf.in2p3.fr

10 Dublin Institute for Advanced Studies, Ireland

11 Landessternwarte, Königstuhl, Heidelberg, Germany

12 Laboratoire de Physique Théorique et Astroparticules, IN2P3/CNRS, Université Montpellier II, France

13 Laboratoire d'Astrophysique de Grenoble, INSU/CNRS, Université Joseph Fourier, France

14 DAPNIA/DSM/CEA, CE Saclay, Gif-sur-Yvette, France
15 Unit for Space Physics, North-West University, Potchefstroom, South Africa

${ }^{16}$ Laboratoire de Physique Nucléaire et de Hautes Energies, IN2P3/CNRS, Universités Paris VI \& VII, France

17 Institute of Particle and Nuclear Physics, Charles University, Prague, Czech Republic

18 Institut für Theoretische Physik, Lehrstuhl IV, Ruhr-Universität Bochum, Germany

19 University of Namibia, Windhoek, Namibia

${ }^{20}$ European Associated Laboratory for Gamma-Ray Astronomy, jointly supported by CNRS and MPG 